\title{
What saccadic eye movements tell us about TMS-induced neuromodulation of the DLPFC and mood changes: a pilot study in bipolar disorders
}

\author{
Lysianne Beynel ${ }^{*}$, Alan Chauvin ${ }^{1}$, Nathalie Guyader ${ }^{2}$, Sylvain Harquel ${ }^{1,3}$, David Szekely ${ }^{4}$, \\ Thierry Bougerol ${ }^{4}$ and Christian Marendaz ${ }^{1}{ }^{*}$ \\ 1 Department of Psychology, Laboratory of Psychology and Neurocognition, Grenoble Alpes University, Université Pierre Mendes France, Grenoble, France \\ 2 Department of Images and Signal, Grenoble Image Parole et Signal Automatique-Lab, Grenoble Alpes University, St Martin d'Héres, Grenoble, France \\ ${ }^{3}$ Department of Psychology, IRMaGe, Grenoble Alpes University, Grenoble, France \\ ${ }^{4}$ Department of Psychiatry and Neurology, Hospital of Grenoble, Grenoble Alpes University, La Tronche, France
}

Edited by:

Olivier A. Coubard, CNS-Fed, France

Reviewed by:

Hao Zhang, Pfizer Neuroscience,

USA

Gregory Kroliczak, Adam Mickiewicz

University in Poznan, Poland

*Correspondence:

Lysianne Beynel and Christian

Marendaz, Department of

Psychology, Laboratory of

Psychology and Neurocognition,

Grenoble Alpes University,

Université Pierre Mendes France,

BP 47, F-38040 Grenoble Cedex 9,

Grenoble, France

e-mail: Lysianne.beynel@

upmf-grenoble.fr;

Christian.Marendaz@upm-

grenoble.fr
The study assumed that the antisaccade (AS) task is a relevant psychophysical tool to assess (i) short-term neuromodulation of the dorsolateral prefrontal cortex (DLPFC) induced by intermittent theta burst stimulation (iTBS); and (ii) mood change occurring during the course of the treatment. Saccadic inhibition is known to strongly involve the DLPFC, whose neuromodulation with iTBS requires less stimulation time and lower stimulation intensity, as well as results in longer aftereffects than the conventional repetitive transcranial magnetic stimulation (rTMS). Active or sham iTBS was applied every day for 3 weeks over the left DLPFC of 12 drug-resistant bipolar depressed patients. To assess the iTBS-induced short-term neuromodulation, the saccadic task was performed just before (S1) and just after (S2) the iTBS session, the first day of each week. Mood was evaluated through Montgomery and Asberg Depression Rating Scale (MADRS) scores and the difference in scores between the beginning and the end of treatment was correlated with AS performance change between these two periods. As expected, only patients from the active group improved their performance from S1 to S2 and mood improvement was significantly correlated with AS performance improvement. In addition, the AS task also discriminated depressive bipolar patients from healthy control subjects. Therefore, the AS task could be a relevant and useful tool for clinicians to assess if the Transcranial magnetic stimulation (TMS)-induced short-term neuromodulation of the DLPFC occurs as well as a "trait vs. state" objective marker of depressive mood disorder.

Keywords: antisaccades, DLPFC, rTMS iTBS, bipolar disorder, short-term neuromodulation, long-term neuromodulation

\section{INTRODUCTION \\ rTMS TREATMENT OF DEPRESSION: NECESSITY TO ASSESS THE INDUCED NEUROMODULATION}

Transcranial magnetic stimulation (TMS) is a non-invasive technique that induces a magnetic field on the skull which changes rapidly enough to induce electrical currents in underlying cortical tissue and thus to induce a neuromodulation effect (Hallet, 2000). Repeated TMS (rTMS) has been used as a therapeutic tool for the treatment of drug-resistant mood disorders since the 1990s; patients receive a daily dose of rTMS over frontal regions for several weeks. The rationale for this treatment is that whereas a single rTMS session induces an early long-term potentiation of the targeted cortical area (short-term neuromodulation), cumulative rTMS sessions induce widespread late long-term potentiation across multiple neural circuits (Noda et al., 2013).

Meta-analyses of rTMS as a treatment for depression have been recently published (Janicak et al., 2010; Slotema et al., 2010;
George and Post, 2011; Berlim et al., 2014). All these studies agree that the therapeutic efficacy of rTMS is statistically significant but affects few patients. For example, Berlim et al. (2014) showed that about $30 \%$ of depressed patients receiving active excitatory rTMS responded to the treatment compared to $10 \%$ of patients who received sham treatment (analysis based on 29 studies using randomized, double-blind and sham-controlled trials).

To improve the therapeutic efficacy of rTMS, a new protocoltheta burst stimulation (TBS) - (Huang et al., 2005) has been developed. TBS has been shown not to differ from rTMS in terms of strength and direction of aftereffects (Thut and Pascual-Leone, 2010; Di Lazzaro et al., 2011) but to exert longer-lasting poststimulation effects (Thut and Pascual-Leone, 2010) with less stimulation time and lower stimulation intensity (Hinder et al., 2014). For these reasons TBS is of particular interest for clinicians, and recently some studies investigated the therapeutic effectiveness of TBS for the treatment of mood disorders, using intermittent theta 
burst stimulation (iTBS) that produces long-term potentiationlike effects, or continuous TBS (cTBS) that produces long-term depression-like effects. Li et al. (2014) compared cTBS, iTBS and sham stimulation. They found that depressive mood improved in all groups, with a better antidepressant effect for iTBS (40\% responders) than for cTBS (25\% responders) and sham $(13 \%$ responders). Plewnia et al. (2014) compared TBS to sham stimulation. They found that $56 \%$ of patients receiving active TBS responded to the treatment compared to $25 \%$ of patients who received sham treatment. As a whole, these results indicate that whatever the TMS protocol, the therapeutic efficacy of rTMS is statistically significant but remains limited, and the rTMS clinical relevance is still debated (Padberg and George, 2009).

Apart from idiopathic reasons, several technical/neurophysiologic factors might account for the lack of TMS efficacy. For example, the stimulation parameters (intensity, frequency) might not be adjusted properly for every patient, the dorsolateral prefrontal cortex (DLPFC) might be identified or targeted incorrectly (Fox et al., 2012), or the TMS treatment might fail to induce short-term neuromodulation of the DLPFC in some patients, which prevents the long-term potentiation. To move forward on these issues, it is crucial to ensure that TMS-induced neuromodulation of the DLPFC effectively occurs. This question requires the development of an instrument that can objectively assess this neuromodulation. To be helpful for clinicians, this instrument has to be non-invasive and easy to use. We assumed that an oculometric task such as the antisaccade (AS) task may provide such a tool (Crevits et al., 2005; Malsert et al., 2012a,b, 2013).

\section{RELEVANCE OF AS TASK TO ASSESS TMS-INDUCED NEUROMODULATION OF THE DLPFC AND OF MOOD IMPROVEMENT}

To perform an AS requires the inhibition of a reflexive saccade toward a target and the generation of a voluntary saccade in the opposite direction (Everling and Fischer, 1998). Several lines of evidence suggest that the DLPFC is involved in a cortical network underlying the inhibition process required to perform a correct AS. Some studies showed that lesions of the DLPFC in humans lead to an increase in inhibition errors during AS tasks (Pierrot-Deseilligny et al., 2003; Ploner et al., 2005). Electrophysiological and TMS studies confirmed the involvement of the DLPFC in saccadic inhibition, suggesting a lateralized inhibitory control of the DLPFC (Müri et al., 1999; Johnston and Everling, 2006; Wegener et al., 2008; Müri and Nyffeler, 2008; but Nyffeler et al., 2007). As a consequence, studying AS performance during rTMS treatment applied over the DLPFC, might inform about the rTMS-induced neuromodulation of this cortical region. Moreover, some studies showed that a clinical improvement could result in cognitive improvement. Biringer et al. (2005) showed that recovery from depression is associated with a recovery of many aspects of executive functions to a normal level. Moreover, using rTMS over the left DLPFC on treatmentresistant depressed patients, Kedzior et al. (2012) found, after 20 days of rTMS treatment, a cognitive improvement for these patients as well as a mood improvement. As a consequence, we expected that the saccadic task could also be a marker of mood improvement.

\section{PILOT STUDY}

To test the relevance of AS task to assess TMS-induced neuromodulation of the DLPFC and of mood improvement, we conducted a pilot study in 12 drug-resistant bipolar depressed patients receiving either active or sham iTBS over the left DLPFC for 1-3 weeks. Short-term TMS-induced neuromodulation was tested by comparing performances to a saccadic task performed just before and just after the iTBS session. It was expected that AS performances would be better just after the iTBS session than before for the iTBS active group and not for the sham group; and that changes in AS performances would be stronger in the contralateral hemifield of the stimulated DLPFC, i.e., here, the right hemifield. The relevance of the AS task to assess patients' mood improvement was analyzed by computing the correlation between mood improvement and AS performance improvement. Besides this, we examined the difference between depressed bipolar patients and healthy controls; it was expected that before treatment, patients' AS performances would be impaired compared to those of healthy subjects.

\section{METHODS}

\section{EXPERIMENTAL DESIGN}

Active or sham iTBS treatment was applied twice a day, 5 days a week for 1-3 weeks. Two sessions of saccadic task were conducted on the first day of each week (D0, D7, and D14), one session (S1) before the iTBS and a second (S2) immediately following the iTBS (Figure 1). To ensure that any improvement in the AS performances could not be accounted for by a simple learning effect, two training sessions were administered 4 days before (D-3) the beginning of the iTBS treatment to minimize the practice-related effects. Two sessions of the saccadic task were also performed at the end of the protocol (D18).

\section{SUBJECTS}

\section{Patient group}

Twelve patients ( 6 females, 6 males; mean age $51.6 \pm 11.7$ years) with drug-resistant bipolar disorder (Types I, II, or III) participated in this randomized double-blind placebo-controlled iTBS study. The study was approved by the regional Ethics Committee (Committee for the Protection of Persons in biomedical researches: CPP Sud-Est VI) and allowed by the ANSM (French National Agency for Medicines and Health Products Safety) (Authorization: 2010-A01085-34).

Patients were randomly assigned to the active or to the sham group. This randomization was performed using a randomization table and assessed 3 by 3 to have the same proportion of patients in each group. As allowed by the protocol of the study, the TMS operator did the un-blinding for the purpose of this study, independently from the clinical research team, who remained fully blind of each patient's treatment status.

The inclusion criteria were having a drug-resistant major depressive episode defined according to DSM IV-TR. The criterion of severity was a score of over 20 with a maximum of 60 on the Montgomery and Asberg Depression Rating Scale (MADRS: Montgomery and Asberg, 1979) (see Table 1 for details). Drug resistance was defined as the absence of a response to any antidepressant treatment over at least a 4 -week period 


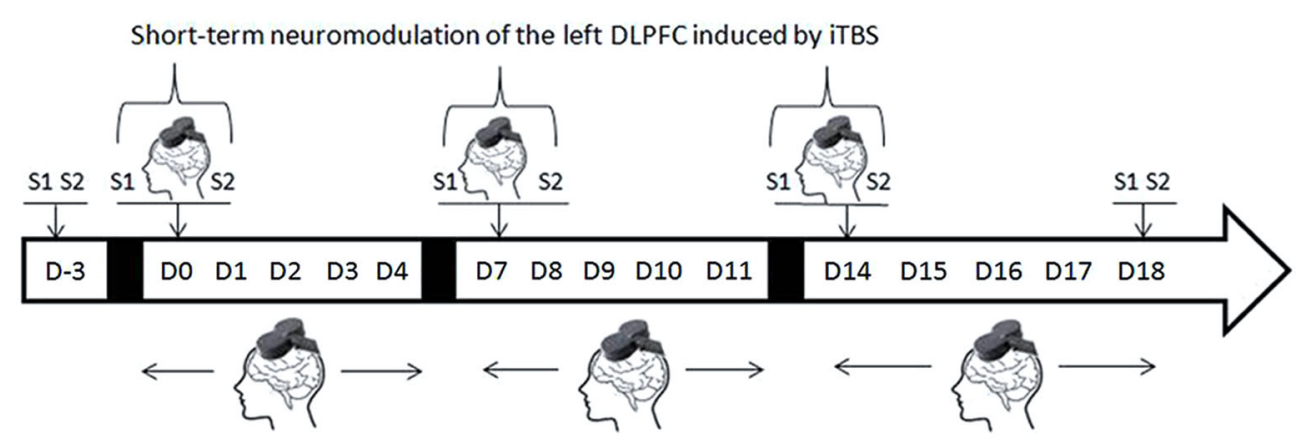

FIGURE 1 | Experimental design ( $D=$ day, S1 and S2 = first and second AS task sessions). On D-3 two AS task sessions are performed for training purposes. Repetitive TMS treatment was administered every day during the week but AS task sessions were performed only on the first day of each week, immediately before (S1) or immediately after (S2) rTMS treatment.
Table 1 | Individual MADRS scores arranged by Group (active vs. sham) and day of treatment (beginning vs. end) and intergroup comparisons.

\begin{tabular}{|c|c|c|c|c|}
\hline Subjects & Group & $\begin{array}{l}\text { MADRS } \\
\text { beginning }\end{array}$ & $\begin{array}{l}\text { MADRS } \\
\text { end }\end{array}$ & Improvement \\
\hline 1 & Active & 30 & 8 & $73.3 \%$ \\
\hline 2 & Active & 36 & 13 & $63.9 \%$ \\
\hline 3 & Active & 38 & 13 & $65.8 \%$ \\
\hline 4 & Active & 32 & 23 & $28.1 \%$ \\
\hline 5 & Active & 24 & 8 & $66.7 \%$ \\
\hline 6 & Sham & 24 & 5 & $79.2 \%$ \\
\hline 7 & Sham & 25 & 16 & $36 \%$ \\
\hline 8 & Sham & 37 & 9 & $75.7 \%$ \\
\hline 9 & Sham & 27 & 4 & $85.2 \%$ \\
\hline 10 & Sham & 32 & 11 & $65.6 \%$ \\
\hline 11 & Sham & 38 & 36 & $5 \%$ \\
\hline 12 & Sham & 25 & 14 & $44 \%$ \\
\hline \multicolumn{5}{|c|}{ Inter-Group Comparisons } \\
\hline & & \multicolumn{2}{|c|}{$(m \pm S D)$} & $t$ and $p$-values \\
\hline \multirow{3}{*}{\multicolumn{2}{|c|}{$\begin{array}{l}\text { Active vs. Sham (beginning) } \\
\text { Active vs. Sham (end) } \\
\text { Active vs. Sham (improvement) }\end{array}$}} & \multirow{3}{*}{\multicolumn{2}{|c|}{$\begin{array}{l}(32 \pm 5 \text { vs. } 30 \pm 6) \\
(13 \pm 6 \text { vs. } 14 \pm 11) \\
(60 \pm 18 \text { vs. } 56 \pm 29)\end{array}$}} & $0.68(0.51)$ \\
\hline & & & & $-0.10(0.92)$ \\
\hline & & & & $0.25(0.81)$ \\
\hline
\end{tabular}

of time. Patients with a history of substance abuse and patients who did not meet inclusion criteria for use of TMS and MRI (no pacemaker, no history of epilepsy or other neurological disorders) were excluded. Mood-stabilizers were allowed during the treatment period only if the patients were stable for at least 4 weeks before the rTMS treatment. Only anxiolytic drugs (cyamemazine or hydroxyzine) at low doses could be administered if necessary during the rTMS treatment period.

\section{Control group}

Twelve control subjects ( 7 females, 5 males; mean age $50.6 \pm$ 10.9 years) also participated in the study. These subjects had no psychiatric history and were not taking any medication. This group only performed one saccadic task session. Every participant provided written, informed consent.

\section{INTERMITTENT THETA BURST STIMULATION (iTBS)}

The iTBS was applied using a figure-of-eight coil (MCF-B65$\mathrm{H0}$ ) and an air-cooled stimulator (MagProX100, MagVenture). For each patient the left DLPFC was delimited using threedimensional magnetic resonance imaging (3D-MRI). The TMS coil was monitored throughout stimulations using a neuronavigation device (TMS Navigator, Localite). We used an iTBS protocol in which a $2 \mathrm{~s}$ train of bursts containing three pulses at $50 \mathrm{~Hz}$ was repeated at $200 \mathrm{~ms}$ (i.e., $5 \mathrm{~Hz}$ ) every $10 \mathrm{~s}$ (Huang et al., 2005), these parameters mimicked the theta rhythm in EEG nomenclature. The TMS operators applied the iTBS twice per day, with a minimum inter-session interval of 3 h every day for 1-3 weeks depending of clinical relevance. For each rTMS session 990 pulses were administered to give a total of $5 \mathrm{~min}$ 30 s of stimulation, thus, patients received 1980 pulses per day. Patients were stimulated with either an active coil (active group: $n=5$ ) or a sham coil (sham group: $n=7$ ). The sham coil made the same "clicking" sound than the active coil, and produced a weak magnetic field on the scalp for reproducing the same skin sensation than the active coil.

We individually set the stimulation intensity at $80 \%$ of the patient's resting motor threshold (RMT), which we determined 3 days before the beginning of the iTBS treatment phase (D-3). We began by placing three electrodes over the patient's first dorsal interosseous muscle (FDI) in a belly-tendon montage. Electromyograms were amplified (1-10 K), band-pass filtered (1-6 KHz), and sampled at $12 \mathrm{KHz}$ using a Dantec Keypoint portable system (Natus Medical Incorporated). We placed the coil on the "hotspot": the position on the motor cortex that elicited the greatest motor-evoked potential (MEP) in the contralateral FDI. We defined the RMT as the minimum stimulation intensity needed to evoke a MEP greater than $50 \mu \mathrm{V}$ on at least 5 out of 10 consecutive trials (Rossini et al., 1994).

\section{SACCADIC TASK}

We used an EyeLink 1000 video-based eye-tracking system (SR Research) with a temporal resolution of $500 \mathrm{~Hz}$. The eye-tracker detects saccades automatically, using three thresholds: velocity $\left(30^{\circ} / \mathrm{s}\right)$, acceleration $\left(8000^{\circ} / \mathrm{s} 2\right)$, and saccadic motion $\left(0.15^{\circ}\right)$. 
Stimuli were displayed on a computer screen located $57 \mathrm{~cm}$ from the participants. The computer screen resolution was $1024 \times$ 768 pixels and the screen refresh rate was $85 \mathrm{~Hz}$. Participants were seated in a darkened room and their heads were stabilized using chin rests. We used a SPAN task (Saccade: Pro, Anti and No) which mixed three types of saccades: antisaccades (AS), nosaccades (NS) and prosaccades (PS). PS was used to verify that the bipolar patients did not suffer from a general deficit in saccadic function. Additionally, compared to a traditional AS task, this mixed saccadic paradigm increased the cognitive load in terms of executive functions, and thus reinforced the implication of the DLPFC (Smith and Jonides, 1999).

Each trial began with a $500 \mathrm{~ms}$ presentation of a white central fixation dot, and then the central fixation dot became red, blue, or green for $2 \mathrm{~s}$. Participants were told to make a PS if it was green, an AS if it was red, or an NS response if it was blue (Figure 2). After this time, a blank screen was displayed for a $200 \mathrm{~ms}$ gap, and a "cue", the number "0", was flashed for $50 \mathrm{~ms}$ at $10^{\circ}$ peripherally (randomly on the right or left side of the screen). During AS trials, patients had to look towards the opposite side from the cue as quickly as possible in order to identify a numeric target ("6" or " 9 "), which was presented for $1 \mathrm{~s}$ beginning as soon as they looked at the correct location (gaze-contingent display) or after a 2-s delay. During PS trials, patients had to look as quickly as possible towards the side of the cue to identify the numeric target. During NS trials, they had to keep their gaze fixed on the center of the screen. There was a break of $1 \mathrm{~s}$ between two successive trials. During the first SPAN session, both patients and healthy controls received 20 practice trials and 80 test trials (16 NS trials, 32 PS trials, and 32 AS trials). In the following SPAN sessions, patients performed 80 test trials. We assessed the performances using the inhibition error rate, i.e., the proportion of saccades towards the cue for AS and NS trials and the latency of correct saccades for PS and AS trials.
We analyzed the oculometric performance, i.e., the inhibition error rates and the saccadic reaction times using Matlab (MATLAB, R2009b, The MathWorks Inc., Natick, MA, 2009) and Statistica (Statistica 10, Statsoft Inc., 1984).

\section{MOOD EVALUATION}

We analyzed MADRS scores at the beginning and at the end of the experiment in order to assess mood changes over the time course of the experiment, as well as to assess responses to the iTBS treatment. We defined "response to the treatment" when there was a $50 \%$ improvement of the MADRS score and "remission" when the MADRS score became lower than 8 .

\section{RESULTS}

In the following results we only present the inhibition error rates in AS trials as we did not find any differences for PS, NS and latencies of AS trials.

\section{COMPARISON OF "HEALTHY SUBJECTS VS. DEPRESSIVE BIPOLAR PATIENTS"}

We performed an ANOVA on inhibition error rates with the Group (bipolar patients $(n=12)$ and healthy controls $(n=12))$ as the between-subjects factor, and the Cue Position (left or right) as the within-subjects factor. This comparison was performed on the learning session only (D-3) for both groups. The ANOVA revealed a main effect of the Group $\left(F_{(1,22)}=4.8 ; p=0.04\right)$. Patients committed significantly more errors than did controls $(26.9 \%$ vs. $13.8 \%)$. We did not find any effect of the Cue Position, nor of the interaction Group $\times$ Cue Position $\left(F_{(1,22)}<1\right)$.

\section{SHORT-TERM ITBS NEUROMODULATION}

We performed another ANOVA on inhibition error rates with the Group" (active $(n=5)$ and sham $(n=7))$ as the betweensubjects factor, and the Session (Session 1 and Session 2) as the within-subjects factor. The ANOVA did not reveal any effect of

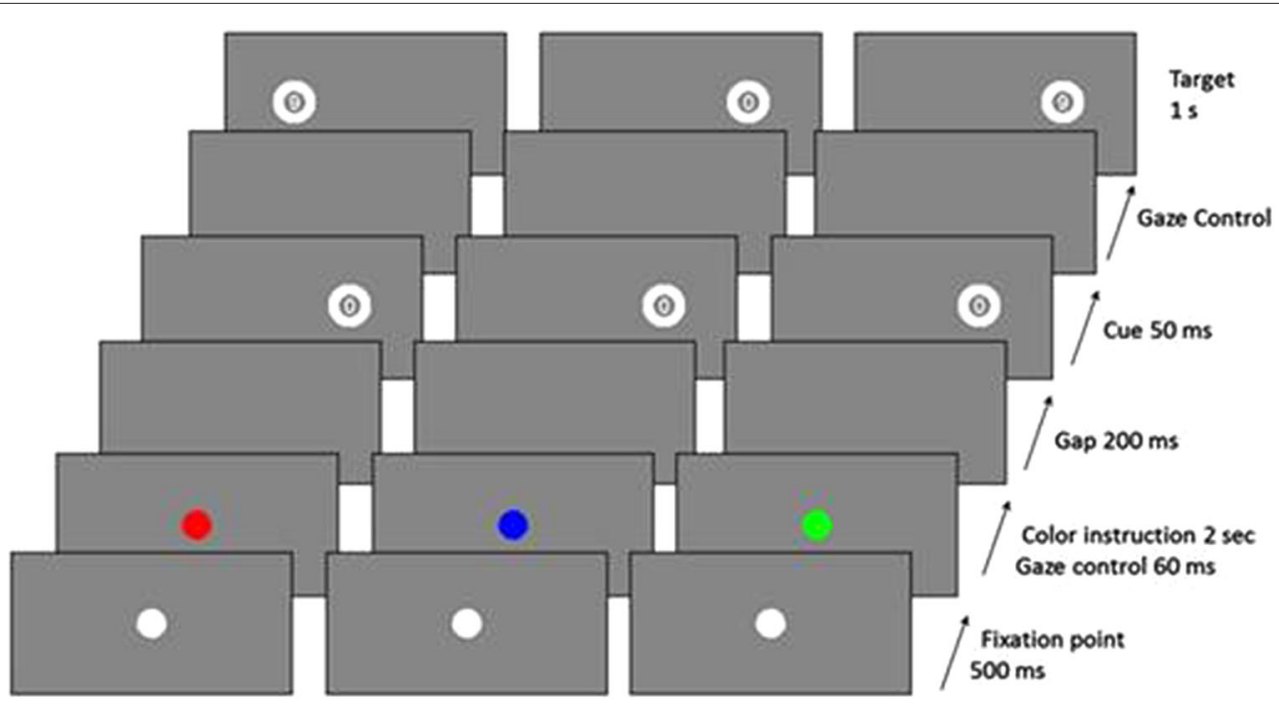

FIGURE 2 | Examples of trial sequences for the three trial types (red = AS, blue = NS, green = PS) (from Malsert et al., 2013) 


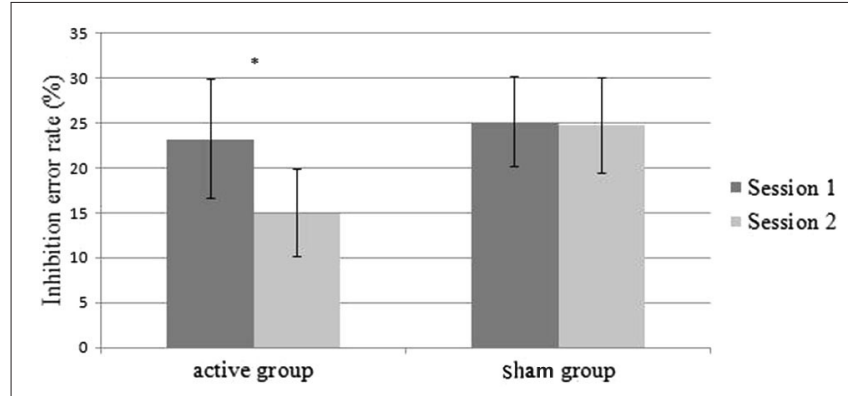

FIGURE 3 | Mean inhibition error rates on the AS task as a function of the sessions (session 1 vs. session 2) and group (active vs. sham).

Errors bars represent standard error.

the Group $\left(F_{(1,10)}<1\right)$. However, we found a main effect of the Session $\left(F_{(1,10)}=6.29 ; p=0.03\right)$. Performances were improved in session 2 compared to session 1 (24.2\% vs. 19.9\%). We also found a significant interaction Group $\times$ Session $\left(F_{(1,10)}=5.29 ; p=0.04\right)$ (Figure 3). Patients in the active group committed fewer errors in session 2 than in session $1(23.2 \%$ vs. $14.9 \%)\left(F_{(1,10)}=9.9 ; p=\right.$ 0.01 ), while the performances of the patients in the sham group did not show any improvements $(25.1 \%$ vs. $24.8 \%)\left(F_{(1,10)}<1\right)$ (Figure 3). When the cue was presented in the right hemifield (contralateral field of iTBS neuromodulation), 100\% of patients in the active group improved their performances (vs. $57 \%$ of the sham group).

\section{EFFECTS OF MOOD IMPROVEMENT}

The MADRS scores analyses did not reveal any differences between the active and sham groups before the treatment ( $p=$ 0.51). After the treatment, four out of five patients in the active group and four out of seven in the sham group responded to the treatment i.e., showed an improvement of more than $50 \%$ on the MADRS scores. The $t$-tests did not reveal any differences between groups, neither on the MADRS scores nor for mood improvement (Table 1). To assess the relevance of the AS task as a marker of this mood improvement, we calculated the Pearson's correlation coefficient between the improvement in the MADRS scores and the difference in inhibition error rates between the end and the beginning of the treatment. We found a significant and positive linear association $\left(r=0.65 ; p=0.02 ; R^{2}=0.42\right)$ : better $\operatorname{mood}$ is associated with better performance in AS (Figure 4).

\section{DISCUSSION}

This study investigated the AS task as a psychophysical tool to assess the short-term neuromodulation that has been hypothesized to be induced by daily iTBS delivered over the left DLPFC. We also examined the ability of the AS task to discriminate depressive bipolar patients from healthy subjects, and to be a marker of mood improvement.

\section{RELEVANCE OF AS TASK TO DISCRIMINATE DEPRESSED BIPOLAR PATIENTS FROM HEALTHY SUBJECTS}

Over the past three decades, there has been an increase in the number of neuropsychophysical studies of saccadic performance

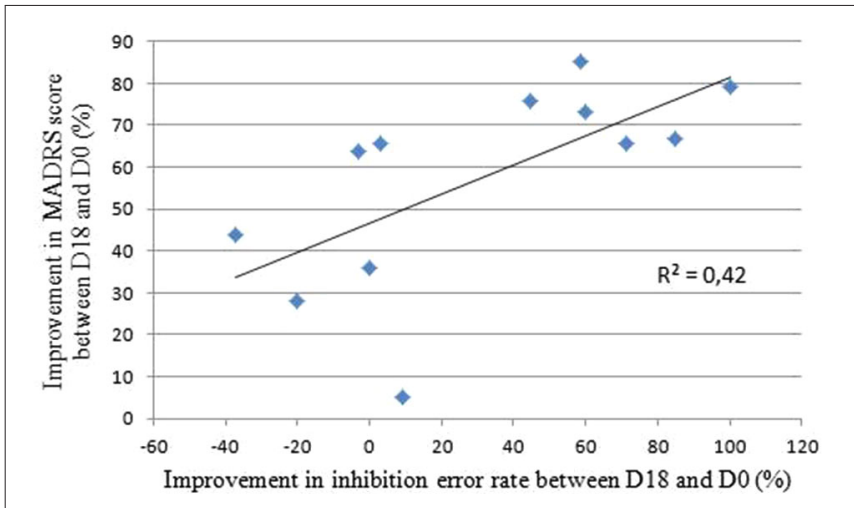

FIGURE 4 | Correlation between the improvements in MADRS scores and inhibition error rate between the end and the beginning of the rTMS treatment. Bravais-Pearson coefficient correlation coefficient equals $R=0.65, p=0.02$ and $R^{2}=0.42$, where $R^{2}$ is the proportion of variance explained by the regression model.

in psychiatric patient groups (Gooding and Basso, 2008). Some authors suggested that performance on the AS task could be used as a psychophysical marker for mood disorders (García-Blanco et al., 2013; Malsert et al., 2013). Our results confirmed that the AS task could discriminate depressed bipolar patients from healthy subjects. We did not find any group differences on PS performance, which means that depressive bipolar patients did not suffer from a general impairment in saccadic function.

Maybe due to the small number of patients, our study did not show group differences associated with the cue position, i.e., no cerebral asymmetry. Whether depressed bipolar patients display a cerebral asymmetry in the inhibitory functions of the DLPFC is still being discussed in the literature (Clark et al., 2006; Savitz and Drevets, 2009); it is a crucial issue since rTMS therapy is often based on the premise that left DLPFC is hypoactive in depression.

\section{RELEVANCE OF AS TASK TO ASSESS SHORT-TERM ITBS-INDUCED NEUROMODULATION OF THE DLPFC}

Until now only one study investigated the AS performance during rTMS treatment applied over the DLPFC to investigate the rTMS neuromodulation effect of this cortical region (Crevits et al., 2005). In their study, the left DLPFC of 11 depressed patients was stimulated with 1000 pulses per day of facilitatory rTMS $(10 \mathrm{~Hz})$. The treatment lasted for at least 10 sessions, with no more than one session a day, over a maximum period of 3 weeks. The AS task was only performed twice: before the first rTMS session and after the last rTMS session. They found a significant decrease in AS latencies at the end of the treatment. However, the absence of a control group of patients receiving sham rTMS prevented the authors from drawing any conclusions about the long-term effects of rTMS. Additionally, the latency reduction in AS might have reflected a practice effect as the AS task was being repeated at the end of the rTMS treatment. Chauvin et al. (2011) studied the evolution of performance across several sessions of an AS task and found that performance only improved (with decreases in AS latency and inhibition error rate) 
over the two first sessions. Finally, mood improvement was able to explain the AS performance improvement (Salvadore et al., 2011).

In our knowledge, our study was the first research investigating by oculometry the short-term iTBS-induced neuromodulation of the DLPFC, using a neuronavigation system. As expected, we observed an aftereffect of the iTBS sessions: only the patients in the active group improved their capacity to inhibit the reflexive saccades immediately after the iTBS sessions. This improvement was consistently observed when the cue was presented in the right hemifield, i.e., processed by the left DLPFC. These results provide evidence that iTBS induces short-term neuromodulation of the targeted cortical area. This means that the AS task could be a useful instrument to ascertain whether short-term neuromodulation induced by iTBS occurs.

\section{RELEVANCE OF AS TASK AS A POTENTIAL MARKER OF MOOD IMPROVEMENT}

We found that the active and sham groups showed a similar mood improvement. This improvement in the sham group and possibly in the active group too was due to a placebo effect (see Mayberg et al., 2002). Some studies showed that mood improvement could result in cognitive improvement. Biringer et al. (2005) showed that recovery from major unipolar depression is associated with a recovery of many aspects of executive functioning, improving executive functioning to a normal level. In accordance with these studies, we found a significant correlation between mood improvement and AS improvement. This indicates that the saccadic task might not only be a useful marker of the short-term neuromodulation, but a marker of mood changes too.

\section{CONCLUSION}

This pilot study investigated the iTBS-induced short-term neuromodulation of the DLPFC. This is a crucial issue since little is known about the aftereffect of TBS over the DLPFC while being used in clinical research, in particular, with psychiatric disorders (Soekadar et al., 2009; Chistyakov et al., 2010; Holzer and Padberg, 2010; Plewnia et al., 2014). Exploring the rTMS/iTBS aftereffects requires the development of an instrument to enable one to objectively measure the short-term TBS-induced neuromodulation, which is the sine qua non condition to long-term neuromodulation taking place (Pascual-Leone et al., 1994; ValeroCabré et al., 2011). Our study demonstrates that an AS task could be used to assess it. Moreover, we confirmed that AS performance could discriminate depressive bipolar patients from healthy subjects and be used as a marker of mood variation (response to treatment or relapse into illness) (Malsert et al., 2012b; Aleman, 2013).

However, due to the small sample size, our findings should be replicated using a larger cohort of patients. Moreover, the oculometric task could be improved by adding an emotional component to increase the load imposed on the DLPFC inhibitory control. Indeed, in humans, the existence of connections between the DLPFC and the limbic regions is well established, although the anatomical details of the connections remain unclear in humans (Fox et al., 2012) and monkeys
(Petrides and Pandya, 1999). Adding emotional cues should improve the psychometric relevance of the oculometric sessions (García-Blanco et al., 2013). Using an implicit emotional oculometric paradigm every day with depressive bipolar patients should enable a finer-grained analysis of short-term neuromodulation induced by rTMS over the DLPFC and could be a way to optimize and customize the TMS treatment by adjusting rTMS parameters of each patient according to the obtained post-effect.

\section{AUTHOR AND CONTRIBUTORS}

Alan Chauvin, Nathalie Guyader, and Christian Marendaz designed the research; Thierry Bougerol and David Szekely analyzed and interpreted clinical data; Lysianne Beynel, Alan Chauvin, Nathalie Guyader, Sylvain Harquel and Christian Marendaz performed research; Lysianne Beynel, Alan Chauvin, Nathalie Guyader, Sylvain Harquel and Christian Marendaz analyzed and interpreted data; Lysianne Beynel, Alan Chauvin, Nathalie Guyader, Sylvain Harquel, Christian Marendaz and Thierry Bougerol wrote the paper; Lysianne Beynel, Alan Chauvin, Nathalie Guyader, Sylvain Harquel, Christian Marendaz and Thierry Bougerol gave the final approval of the version to be published.

Lysianne Beynel, Alan Chauvin, Nathalie Guyader, Sylvain Harquel, Christian Marendaz, David Szekely and Thierry Bougerol agree to be accountable for all aspects of the work in ensuring that questions related to the accuracy or integrity of any part of the work are appropriately investigated and resolved.

\section{ACKNOWLEDGMENTS}

This work was supported by Grenoble-Alpes University, the National Center for Scientific Research (CNRS), Rhône-Alpes Region, Health and Society research association. The authors want to thank Marcia Bécu for her experimental help; Vincent Meille and Benoit Trojak for the DLPFC localization.

\section{REFERENCES}

Aleman, A. (2013). Use of repetitive transcranial magnetic stimulation for treatment in psychiatry. Clin. Psychopharmacol. Neurosci. 11, 53-59. doi: 10.9758/ cpn.2013.11.2.53

Berlim, M. T., van den Eynde, F., Tovar-Perdomo, S., and Daskalakis, Z. J. (2014). Response, remission and drop-out rates following high-frequency repetitive transcranial magnetic stimulation (rTMS) for treating major depression: a systematic review and meta-analysis of randomized, double-blind and sham-controlled trials. Psychol. Med. 44, 225-239. doi: 10.1017/s0033291713 000512

Biringer, E., Lundervold, A., Stordal, K., Mykletun, A., Egeland, J., Bottlender, R., et al. (2005). Executive function improvement upon remission of recurrent unipolar depression. Eur. Arch. Psychiatry Clin. Neurosci. 255, 373-380. doi: 10. 1007/s00406-005-0577-7

Chauvin, A., Guyader, N., Braun, M. N., Quetard, B., and Marendaz, C. (2011). Reliability and sensitivity of antisaccade in a block versus mixed paradigm. Vis. Sci. Soc. 11:551. doi: 10.1167/11.11.551

Chistyakov, A. V., Rubicsek, O., Kaplan, B., Zaaroor, M., and Klein, E. (2010). Safety, tolerability and preliminary evidence for antidepressant efficacy of theta-burst transcranial magnetic stimulation in patients with major depression. Int. J. Neuropsychopharmacol. 13, 387-393. doi: 10.1017/s1461145710 000027

Clark, C. P., Brown, G. G., Archibald, S. L., Fennema-Notestine, C., Braun, D. R., Thomas, L. S., et al. (2006). Does amygdalar perfusion correlate with 
antidepressant response to partial sleep deprivation in major depression? Psychiatry Res. 146, 43-51. doi: 10.1016/j.pscychresns.2005.09.007

Crevits, L., van den Abbeele, D., Audenaert, K., Goethals, M., and Dierick, M. (2005). Effect of repetitive transcranial magnetic stimulation on saccades in depression: a pilot study. Psychiatry Res. 135, 113-119. doi: 10.1016/j.psychres. 2003.10.008

Di Lazzaro, V., Dileone, M., Pilato, F., Capone, F., Musumeci, G., Ranieri, F., et al. (2011). Modulation of motor cortex neuronal networks by rTMS: comparison of local and remote effects of six different protocols of stimulation. J. Neurophysiol. 105, 2150-2156. doi: 10.1152/jn.00781.2010

Everling, S., and Fischer, B. (1998). The antisaccade: a review of basic research and clinical studies. Neuropsychologia 36, 885-899. doi: 10.1016/s00283932(98)00020-7

Fox, M. D., Buckner, R. L., White, M. P., Greicius, M. D., and Pascual-Leone, A. (2012). Efficacy of transcranial magnetic stimulation targets for depression is related to intrinsic functional connectivity with the subgenual cingulate. Biol. Psychiatry 72, 595-603. doi: 10.1016/j.biopsych.2012.04.028

García-Blanco, A. C., Perea, M., and Salmerón, L. (2013). Attention orienting and inhibitory control across the different mood states in bipolar disorder: an emotional antisaccade task. Biol. Psychol. 94, 556-561. doi: 10.1016/j.biopsycho. 2013.10.005

George, M. S., and Post, R. M. (2011). Daily left prefrontal repetitive transcranial magnetic stimulation for acute treatment of medication-resistant depression. Am. J. Psychiatry 168, 356-364. doi: 10.1176/appi.ajp.2010.100 60864

Gooding, D. C., and Basso, M. A. (2008). The tell-tale tasks: a review of saccadic research in psychiatric patient populations. Brain Cogn. 68, 371-390. doi: 10. 1016/j.bandc.2008.08.024

Hallet, M. (2000). Transcranial magnetic stimulation and the human brain. Nature 406, 147-150. doi: 10.1038/35018000

Hinder, M. R., Goss, E. L., Fujiyama, H., Canty, A. J., Garry, M. I., Rodger, J., et al. (2014). Inter- and intra-individual variability following intermittent theta burst stimulation: implications for rehabilitation and recovery. Brain Stimul. 7, 365371. doi: 10.1016/j.brs.2014.01.004

Holzer, M., and Padberg, F. (2010). Intermittent theta burst stimulation (iTBS) ameliorates therapy-resistant depression: a case series. Brain Stimul. 3, 181-183. doi: 10.1016/j.brs.2009.10.004

Huang, Y. Z., Edwards, M. J., Rounis, E., Bhatia, K. P., and Rothwell, J. C. (2005). Theta burst stimulation of the human motor cortex. Neuron 45, 201-206. doi: 10.1016/j.neuron.2004.12.033

Janicak, P. G., Nahas, Z., Lisanby, S. H., Solvason, H. B., Sampson, S. M., McDonald, W. M., et al. (2010). Durability of clinical benefit with transcranial magnetic stimulation (TMS) in the treatment of pharmacoresistant major depression: assessment of relapse during a 6-month, multisite, open-label study. Brain Stimul. 3, 187-199. doi: 10.1016/j.brs.2010.07.003

Johnston, K., and Everling, S. (2006). Monkey dorsolateral prefrontal cortex sends task-selective signals directly to the superior colliculus. J. Neurosci. 26, 1247112478. doi: 10.1523/jneurosci.4101-06.2006

Kedzior, K. K., Rajput, V., Price, G., Lee, J., and Martin-Iverson, M. (2012). Cognitive correlates of repetitive transcranial magnetic stimulation (rTMS) in treatment-resistant depression-a pilot study. BMC Psychiatry 12:163. doi: 10. 1186/1471-244X-12-163

Li, C. T., Chen, M. H., Juan, C. H., Huang, H. H., Chen, L. F., Hsieh, J. C., et al. (2014). Efficacy of prefrontal theta-burst stimulation in refractory depression: a randomized sham-controlled study. Brain 137, 2088-2098. doi: 10. 1093/brain/awu109

Malsert, J., Chauvin, A., Guyader, N., Polosan, M., Szekely, D., Bougerol, T., et al. (2013). Saccadic performance and cortical excitability as trait-markers and statemarkers in rapid cycling bipolar disorder: a two-case follow-up study. Front. Psychiatry 3:112. doi: 10.3389/fpsyt.2012.00112

Malsert, J., Guyader, N., Chauvin, A., and Marendaz, C. (2012a). Having to identify a target reduces antisaccade latencies in mixed saccadic paradigms: a top-down effect released by tonic prefrontal activation? Cogn. Neurosci. 3, 105-111. doi: 10. 1080/17588928.2012.666965

Malsert, J., Guyader, N., Chauvin, A., Polosan, M., Poulet, E., Szekely, D., et al. (2012b). Antisaccades as a follow-up tool in major depressive disorder therapies: a pilot study. Psychiatry Res. 200, 1051-1053. doi: 10.1016/j.psychres.2012. 05.007
Mayberg, H. S., Silva, J. A., Brannan, S. K., Tekell, J. L., Mahurin, R. K., McGinnis, S., et al. (2002). The functional neuroanatomy of the placebo effect. Am. J. Psychiatry 159, 728-737. doi: 10.1176/appi.ajp.159.5.728

Montgomery, S. A., and Asberg, M. (1979). A new depression scale designed to be sensitive to change. Br. J. Psychiatry 134, 382-389. doi: 10.1192/bjp.134. 4.382

Müri, R. M., and Nyffeler, T. (2008). Neurophysiology and neuroanatomy of reflexive and volitional saccades as revealed by lesion studies with neurological patients and transcranial magnetic stimulation (TMS). Brain Cogn. 68, 284-292. doi: 10.1016/j.bandc.2008.08.018

Müri, R. M., Rivaud, S., Gaymard, B., Ploner, C. J., Vermersch, A. I., Hess, C. W., et al. (1999). Role of the prefrontal cortex in the control of express saccades. A transcranial magnetic stimulation study. Neuropsychologia 37, 199-206. doi: 10. 1016/s0028-3932(98)00094-3

Noda, Y., Nakamura, M., Saeki, T., Inoue, M., Iwanari, H., and Kasai, K. (2013). Potentiation of quantitative electroencephalograms following prefrontal repetitive transcranial magnetic stimulation in patients with major depression. Neurosci. Res. 77, 70-77. doi: 10.1016/j.neures.2013. 06.002

Nyffeler, T., Müri, R. M., Bucher-Ottiger, Y., Pierrot-Deseilligny, C., Gaymard, B., and Rivaud-Pechoux, S. (2007). Inhibitory control of the human dorsolateral prefrontal cortex during the anti-saccade paradigm a transcranial magnetic stimulation study. Eur. J. Neurosci. 26, 1381-1385. doi: 10.1111/j.1460-9568. 2007.05758.x

Padberg, F., and George, M. S. (2009). Repetitive transcranial magnetic stimulation of the prefrontal cortex in depression. Exp. Neurol. 219, 2-13. doi: 10.1016/j. expneurol.2009.04.020

Pascual-Leone, A., Valls-Solé, J., Wassermann, E. M., and Hallet, M. (1994). Responses to rapid-rate transcranial magnetic stimulation of the human motor cortex. Brain 117, 847-858. doi: 10.1093/brain/117.4.847

Petrides, M., and Pandya, D. N. (1999). Dorsolateral prefrontal cortex: comparative cytoarchitectonic analysis in the human and the macaque brain and corticocortical connection patterns. Eur. J. Neurosci. 11, 1011-1036. doi: 10.1046/j.14609568.1999.00518.x

Pierrot-Deseilligny, C., Müri, R. M., Ploner, C. J., Gaymard, B., Demeret, S., and Rivaud-Pechoux, S. (2003). Decisional role of the dorsolateral prefrontal cortex in ocular motor behaviour. Brain 126, 1460-1473. doi: 10.1093/brain/ awg148

Plewnia, C., Pasqualetti, P., Große, S., Schlipf, S., Wasserka, B., Zwissler, B., et al. (2014). Treatment of major depression with bilateral theta burst stimulation: a randomized controlled pilot trial. J. Affect. Disord. 156, 219-223. doi: 10.1016/j. jad.2013.12.025

Ploner, C. J., Gaymard, B. M., Rivaud-Péchoux, S., and Pierrot-Deseilligny, C. (2005). The prefrontal substrate of reflexive saccade inhibition in humans. Biol. Psychiatry 57, 1159-1165. doi: 10.1016/j.biopsych.2005.02.017

Rossini, P. M., Barker, A. T., Berardelli, A., Caramia, M. D., Caruso, G., Cracco, R. Q., et al. (1994). Non-invasive electrical and magnetic stimulation of the brain, spinal cord and roots: basic principles and procedures for routine clinical application. Report of an IFCN committee. Electroencephalogr. Clin. Neurophysiol. 91, 79-92. doi: 10.1016/0013-4694(94)90029-9

Salvadore, G., Nugent, A. C., Lemaitre, H., Luckenbaugh, D. A., Tinsley, R., Cannon, D. M., et al. (2011). Prefrontal cortical abnormalities in currently depressed versus currently remitted patients with major depressive disorder. Neuroimage 54, 2643-2651. doi: 10.1016/j.neuroimage.2010. 11.011

Savitz, J., and Drevets, W. C. (2009). Bipolar and major depressive disorder: neuroimaging the developmental-degenerative divide. Neurosci. Biobehav. Rev. 33, 699-771. doi: 10.1016/j.neubiorev.2009.01.004

Slotema, C. W., Blom, J. D., Hoek, H. W., and Sommer, I. E. (2010). Should we expand the toolbox of psychiatric treatment methods to include Repetitive Transcranial Magnetic Stimulation (rTMS)? A meta-analysis of the efficacy of rTMS in psychiatric disorders. J. Clin. Psychiatry 71, 873-884. doi: 10.4088/JCP. 08m04872gre

Smith, E. E., and Jonides, J. (1999). Storage and executive processes in the frontal lobes. Science 283, 1657-1661. doi: 10.1126/science.283.5408.1657

Soekadar, S. R., Arfeller, C., Rilk, A., Plontke, S. K., and Plewnia, C. (2009). Theta burst stimulation in the treatment of incapacitating tinnitus accompanied by severe depression. CNS Spectr. 14, 208-211. 
Thut, G., and Pascual-Leone, A. (2010). A review of combined TMS-EEG studies to characterize lasting effects of repetitive TMS and assess their usefulness in cognitive and clinical neuroscience. Brain Topogr. 22, 219-232. doi: 10. 1007/s10548-009-0115-4

Valero-Cabré, A., Pascual-Leone, A., and Coubard, O. (2011). La stimulation magnétique transcrânienne (SMT) dans la recherche en neuroscience. Rev. Neurol. 167, 291-316. doi: 10.1016/j.neurol.2010.10.013

Wegener, S. P., Johnston, K., and Everling, S. (2008). Microstimulation of monkey dorsolateral prefrontal cortex impairs antisaccade performance. Exp. Brain Res. 190, 463-473. doi: 10.1007/s00221-008-1488-4

Conflict of Interest Statement: The authors declare that the research was conducted in the absence of any commercial or financial relationships that could be construed as a potential conflict of interest.
Received: 04 February 2014; accepted: 31 July 2014; published online: 19 August 2014.

Citation: Beynel L, Chauvin A, Guyader N, Harquel S, Szekely D, Bougerol T and Marendaz C (2014) What saccadic eye movements tell us about TMS-induced neuromodulation of the DLPFC and mood changes: a pilot study in bipolar disorders. Front. Integr. Neurosci. 8:65. doi: 10.3389/fnint.2014.00065

This article was submitted to the journal Frontiers in Integrative Neuroscience.

Copyright () 2014 Beynel, Chauvin, Guyader, Harquel, Szekely, Bougerol and Marendaz. This is an open-access article distributed under the terms of the Creative Commons Attribution License (CC BY). The use, distribution or reproduction in other forums is permitted, provided the original author(s) or licensor are credited and that the original publication in this journal is cited, in accordance with accepted academic practice. No use, distribution or reproduction is permitted which does not comply with these terms. 